\title{
High-Frequency Stimulation of the Subthalamic Nucleus Blocks Compulsive-Like Re-Escalation of Heroin Taking in Rats
}

\author{
Carrie L Wade*,', Marsida Kallupi', Daniel O Hernandez', Emmanuel Breysse',2, Giordano de Guglielmo', \\ Elena Crawford', George F Koob', Paul Schweitzer', Christelle Baunez ${ }^{2}$ and Olivier George ${ }^{*, I}$ \\ 'Committee on the Neurobiology of Addictive Disorders, The Scripps Research Institute, La Jolla, CA, USA; ${ }^{2}$ CNRS and Aix Marseille Université, INT \\ UMR7289, Marseille, France
}

\begin{abstract}
Opioid addiction, including addiction to heroin, has markedly increased in the past decade. The cost and pervasiveness of heroin addiction, including resistance to recovery from addiction, provide a compelling basis for developing novel therapeutic strategies. Deep brain stimulation may represent a viable alternative strategy for the treatment of intractable heroin addiction, particularly in individuals who are resistant to traditional therapies. Here we provide preclinical evidence of the therapeutic potential of high-frequency stimulation of the subthalamic nucleus (STN HFS) for heroin addiction. STN HFS prevented the re-escalation of heroin intake after abstinence in rats with extended access to heroin, an animal model of compulsive heroin taking. STN HFS inhibited key brain regions, including the substantia nigra, entopeduncular nucleus, and nucleus accumbens shell measured using brain mapping analyses of immediate-early gene expression and produced a robust silencing of STN neurons as measured using whole-cell recording ex vivo. These results warrant further investigation to examine the therapeutic effects that STN HFS may have on relapse in humans with heroin addiction.

Neuropsychopharmacology (2017) 42, 1850-1859; doi:I0.1038/npp.2016.270; published online I February 2017
\end{abstract}

\section{INTRODUCTION}

Opioid addiction, including addiction to heroin, oxycodone, and other opioids, has markedly increased in the past decade. According to the National Institute on Drug Abuse, an estimated 4.2 million people $(1.6 \%$ of the population in the United States) in 2011 had tried opioids at some time in their lives, with $23 \%$ converting to an opioid use disorder. Emergency department visits due to the nonmedical use of prescription opioids has increased from 168000 in 2004 to 366000 in 2011 (Crane, 2015). Individuals with heroin addiction lose an average of 18 years of potential life before age 65 years because of heroin overdose, chronic liver disease, or accidents (Smyth et al, 2007). Current treatments include cognitive behavioral therapy, replacement therapy (eg, methadone and buprenorphine), and antagonist therapy (eg, naltrexone). However, of this population, $40-60 \%$ will relapse following traditional treatment and rehabilitation within a year (McLellan et al, 2000). The resistance to treatment and recidivism of many opioid-addicted individuals provide a compelling basis for developing novel therapeutic strategies. It is estimated that untreated heroin

*Correspondence: Dr CL Wade, Department of Neuroscience, University of Minnesota, 32I Church St SE, Minneapolis, MN 55455 USA or Dr O George, Committee on the Neurobiology of Addictive Disorders, The Scripps Research Institute, 10550 North Torrey Pines Road, SP30-2400, La Jolla, CA 92037, USA, Tel: 858784 8030, Fax: 858784 7405, E-mail: wade0040@umn.edu or ogeorge@scripps.edu Received 12 October 2016; revised 17 November 2016; accepted 22 November 2016; accepted article preview online 5 December 2016 addiction costs society up to US $\$ 20,000$ per year per individual, and recent estimates show that deep brain stimulation (DBS) may be a cost-effective alternative for the treatment of addiction, particularly in patients with intractable heroin addiction (Stephen et al, 2012). Interest in using DBS to manage and treat neurological and psychiatric disorders, such as Parkinson's disease, obsessive compulsive disorder, severe depression, and recently drug addiction, is increasing because of both its symptom management and external control of the stimulation (Hamani et al, 2014). However, little work has explored the efficacy of highfrequency stimulation (HFS) in animal models of compulsive-like drug use. In human studies, only one brain structure, the nucleus accumbens, has been evaluated as a potential target (Kuhn et al, 2014; Valencia-Alfonso et al, 2012; Zhou et al, 2011). Extending this finding to animal models, it has been demonstrated that the shell portion but not the core of the nucleus accumbens provides the most therapeutic efficacy on cocaine seeking and reinstatement (Guercio et al, 2015; Guo et al, 2013; Vassoler et al, 2008, 2013). Therefore, there is a critical need to identify novel brain targets and provide preclinical evidence of the therapeutic efficacy of DBS for heroin addiction.

Converging evidence suggests that the subthalamic nucleus (STN) may be a promising new target. Patients who are treated with DBS for Parkinson's disease, including STN HFS, can also be relieved from obsessive compulsive disorder (Mallet et al, 2002) or from other compulsive behaviors, including gambling and compulsive shopping (Ardouin $e t$ al, 2006; Witjas et al, 2005) that were evident before diagnosis of 
Parkinson's disease. Moreover, one clinical study showed that OCD patients naïve to L-DOPA treatment had relief from compulsive symptoms (Mallet et al, 2008) and that rat studies have shown that lesions of the STN and STN HFS decrease the motivation for cocaine (Baunez et al, 2005; Pelloux et al, 2014; Rouaud et al, 2010). However, unknown is whether STN HFS exerts a therapeutic effect in a preclinical model of compulsive-like heroin taking.

In the present study, we tested the hypothesis that STN HFS decreases the re-escalation of heroin self-administration after abstinence in rats using an animal model of compulsive-like heroin taking. We used two animal models of heroin intake. First, we examined the effect of STN HFS on heroin self-administration and progressive-ratio (PR) responding in rats with short access (ShA) to heroin selfadministration for $3 \mathrm{~h}$, a model that is associated with modest escalation of heroin intake (Chen et al, 2006; Vendruscolo et al, 2011). Second, we examined the effect of STN HFS on heroin self-administration in rats with long access (LgA) to heroin self-administration for $12 \mathrm{~h}$, a model that is associated with higher escalation of heroin intake and the emergence of additional physical and motivational markers of addiction, including pronounced re-escalation of heroin intake after abstinence (Chen et al, 2006; Schlosburg et al, 2013; Vendruscolo et al, 2011). We also examined the consequences of STN HFS on the neuronal network associated with the STN and addiction-related brain regions using immediate-early gene brain mapping techniques and whole-cell recordings.

\section{MATERIALS AND METHODS}

\section{Subjects}

Adult male Wistar rats (total $n=32, n=5-9$ per group; Charles River, Raleigh, NC, USA), weighing $225-275 \mathrm{~g}$ at the beginning of the experiments, were housed in groups of 2-3 per cage in a temperature-controlled $\left(22^{\circ} \mathrm{C}\right)$ vivarium on a $12 \mathrm{~h} / 12 \mathrm{~h}$ light-dark cycle (lights on at 0600 hours) with ad libitum access to food and water. The animals were allowed to acclimate to the animal facilities for at least 7 days before surgery. All of the procedures adhered to the National Institutes of Health Guide for the Care and Use of Laboratory Animals and were approved by the Institutional Animal Care and Use Committee of The Scripps Research Institute.

\section{Drugs}

Heroin $(0.06 \mathrm{mg} / \mathrm{kg}$ per infusion) was obtained from the United States National Institute on Drug Abuse and dissolved in $0.9 \%$ saline.

\section{Surgery}

The rats were anesthetized with isoflurane (1.5-2.5\%) and prepared with chronic intravenous silastic catheters (Dow Corning, Midland, MI, USA) in the right jugular vein. The catheter was secured to the vein with suture thread and passed subcutaneously to exit dorsally on the animal's back. After surgery, the catheters were flushed daily with $0.2 \mathrm{ml}$ of a sterile antibiotic solution that contained heparinized saline (30 USP units $/ \mathrm{ml}$ ) and the antibiotic cefazolin. The rats were allowed to recover for 7 days before behavioral testing (Figure 1a). As described previously (Rouaud et al, 2010), electrodes that were made of two Teflon-insulated platinum wires $(125 \mu \mathrm{m}$ diameter) were implanted bilaterally in the STN. The electrodes were implanted at the following coordinates (Paxinos et al, 1985) from bregma: anterior/ posterior, $-3.7 \mathrm{~mm}$, lateral, $\pm 2.4 \mathrm{~mm}$, dorsal/ventral, $-8.3 \mathrm{~mm}$ (from skull), and incisor bar set at $-3.3 \mathrm{~mm}$. Control animals were implanted with electrodes that were not stimulated. See Figures $1 b$ and $c$ for a representative example of the most dorsal location of electrodes in the STN.
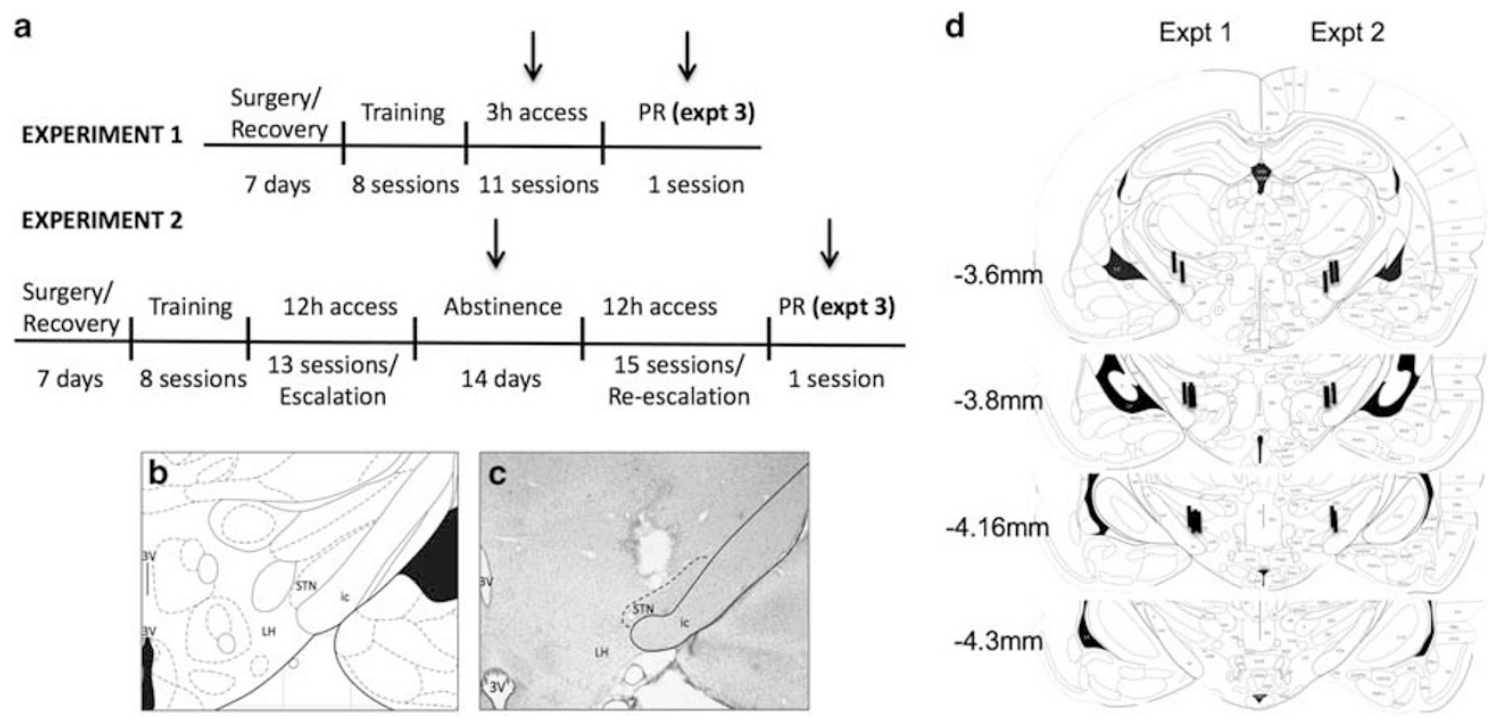

Figure I Experimental design. (a) Experimental timeline for Experiments (Expt) I-3. Arrows indicate periods of STN HFS. Note that control animals were implanted with stimulating electrodes but were not stimulated to control for the effect of the surgery. (b) Brain atlas. (c) Representative image of the tip of the electrode using Cresyl violet staining. (d) Location of electrodes in Experiment I (left) and Experiment 2 (right). ic, internal capsule; LH, lateral hypothalamus; STN, subthalamic nucleus; 3V, third ventricle. 


\section{Self-Administration Chambers}

Self-administration sessions were conducted in standard operant chambers (Med Associates, St Albans, VT, USA). The chambers $(30.5 \mathrm{~cm} \times 24.1 \mathrm{~cm} \times 29.2 \mathrm{~cm})$ were located in a dimly lit room and individually enclosed in wooden cubicles that were fitted with a ventilation fan that also muted external noise. Each operant chamber had two opaque panels as the right and left walls and two clear Plexiglas panels at the front and back walls. The floor consisted of $6 \mathrm{~mm}$ diameter steel bars that were spaced $15 \mathrm{~mm}$ apart. Two retractable levers $(2 \mathrm{~cm} \times 4 \mathrm{~cm} \times 0.3 \mathrm{~cm})$ were mounted $7 \mathrm{~cm}$ above the grid floor on the right operant panel. A white light diode was mounted $8.5 \mathrm{~cm}$ above each lever. A $1.1 \mathrm{~W}$ miniature light bulb that was synchronized to the vivarium's light/dark cycle illuminated the chamber. A spring-covered Tygon tube connected the animal's catheter through a fluid swivel to a syringe $(50 \mathrm{ml})$ that contained heroin. The syringe was placed inside a syringe pump (Razel) that was placed outside and above the chamber. A computer controlled the delivery of fluids and presentation of visual stimuli and recorded the behavioral data.

\section{Deep Brain Stimulation}

Based on previous studies, the stimulation was set at a $130 \mathrm{~Hz}$ frequency and $60 \mu \mathrm{s}$ pulse width (Benazzouz et al, 1995; Darbaky et al, 2003). The intensity was set for each individual rat on the first day of behavioral testing, $10 \mu \mathrm{A}$ below the threshold of induction of hyperkinetic movements of the contralateral paw $(50-130 \mu \mathrm{A})$, consistent with previous studies (Baunez et al, 2005; Darbaky et al, 2003; Rouaud et al, 2010). Animals that did not display hyperkinetic movements when setting the individual intensity, indicating that the electrodes were misplaced, were excluded from the study. Two rats were excluded from analysis in study 1 and three rats were excluded from analysis in study 2 owing to the lack of hyperkinetic movements. All animals in studies 1 and 2 had confirmed electrode localization within the STN upon extraction, and locations of each electrode in experiments 1 (left) and 2 (right) are represented in Figure 1d. Bilateral STN HFS was applied throughout the self-administration session.

\section{Self-Administration Procedures}

Before each self-administration session, the catheters were flushed with $0.2 \mathrm{ml}$ of a sterile antibiotic solution that contained heparinized saline (30 USP units/ml) and the antibiotic cefazolin $(160 \mathrm{mg} / \mathrm{ml})$. Both the training and escalation phases followed a fixed-ratio 1 (FR1) schedule of reinforcement, in which every lever press was reinforced with an intravenous infusion of heroin $(0.1 \mathrm{ml}, 60 \mu \mathrm{g} / \mathrm{kg}$ per infusion). Each infusion was followed by a $5 \mathrm{~s}$ timeout period, during which lever presses did not result in an infusion. The animals were allowed to self-administer in 3 or $12 \mathrm{~h}$ sessions 5 days per week. During the total duration of the sessions, the animals were given ad libitum access to food and water. Following each session, the animals were returned to their home cages.

Experiment 1 (Figure 1a): The animals (control $n=9$; DBS $n=11$ ) were trained to self-administer heroin until stable responding occurred. Following 3 days of stable responding, stimulated and non-stimulated control animals were allowed to self-administer heroin in daily $3 \mathrm{~h}$ sessions. Note that control animals were implanted with stimulating electrodes but were not stimulated.

Experiment 2 ( Figure 1a): A separate group of animals ( $n=8$ per group) was trained to self-administer heroin in daily $12 \mathrm{~h}$ sessions during the dark/active cycle, showing escalation of heroin intake over the course of the experiment (14 sessions). After the first escalation, the animals had no access to heroin for 2 weeks. Following these 2 weeks of abstinence, the animals were allowed to self-administer heroin in $12 \mathrm{~h}$ sessions according to the re-escalation protocol (15 sessions). The animals were separated into two groups: one group received stimulation for the duration of the self-administration session and one group did not. The animals in each group were matched for heroin intake. Additionally, we tested the reversibility of STN HFS by allowing the animals to self-administer heroin following 2 days of no stimulation. Note that control animals were implanted with stimulating electrodes but were not stimulated.

Experiment 3 (Figure 1a): Following the last day of selfadministration in the 3 and $12 \mathrm{~h}$ sessions, the animals were tested for breakpoints on a PR schedule of reinforcement. The rats were given 1 day off after their final escalation session on an FR1 schedule and then tested in a 6-h PR session as described previously (Barbier et al, 2013). Each successive infusion required consecutively greater leverpressing requirements, with the following PR schedule, in which the response requirement increased by steps of 1 up to 50 lever presses and then by steps of 10 , thereafter: $1,1,2,2$, $3,3, \ldots, 50,60,70,80,90,100$. In this test, the response requirement for the next reinforcer increased progressively, and the breakpoint at which the animals stopped working for the dose of drug was taken as an index of motivation.

\section{Locomotor Activity Assessment}

Locomotor activity was assessed in rats 2 weeks following the last self-administration (re-escalation) session. The operant chambers were divided into four parts, and crossings between each quadrant were counted for $30 \mathrm{~min}$. Section breaks were counted for $15 \mathrm{~min}$ without stimulation. The stimulation was then turned on, and section breaks were counted for $15 \mathrm{~min}$ during stimulation. In addition, lever pressing on the inactive lever was recorded during the reescalation phase and compared between groups. For all of these measures, stimulated animals were compared with control, non-stimulated animals.

\section{Fos Immunohistochemisty}

To evaluate alterations in neuronal activity, we examined the expression of the immediate-early gene c-fos in animals naïve to heroin. A separate group of rats ( $n=5 /$ group) was implanted with electrodes and stimulated for $30 \mathrm{~min}$. Sixty minutes following stimulation, the animals (along with nonstimulated controls) were killed and perfused for immunohistochemistry (IHC). Following administration of 35\% (w/ v) chloral hydrate $(2 \mathrm{ml} / \mathrm{kg}, 70 \mathrm{mg} / \mathrm{kg})$, the animals were transcardially perfused, first with saline and then with a $4 \%$ 
paraformaldehyde solution. Brains were postfixed at $4{ }^{\circ} \mathrm{C}$ in paraformaldehyde for 6 days following extraction and cryoprotected in $30 \%$ sucrose for 3 weeks before freezing at $-80^{\circ} \mathrm{C}$. The brains were then sectioned into $40 \mu \mathrm{m}$ slices and preserved in phosphate-buffered saline (PBS) that contained $1 \%$ sodium azide. All of the experimental groups were processed in parallel using the same immunohistochemical procedures. Free-floating sections were rinsed in PBS, incubated for $30 \mathrm{~min}$ in $1 \%$ hydrogen peroxide PBS solution to quench endogenous peroxidase activity, rinsed several times in PBS, and incubated in a blocking solution that contained $0.1 \%$ bovine serum albumin (BSA), 5\% normal donkey serum, and $0.3 \%$ Triton X-100 in PBS for $60 \mathrm{~min}$. The sections were incubated in rabbit anti-c-fos polyclonal antibody diluted 1:2500 (Cell Signaling) in PBS that contained $0.5 \%$ Tween-20,0.1\% BSA, and 5\% normal donkey serum for $24 \mathrm{~h}$ at $4{ }^{\circ} \mathrm{C}$. After incubation in the primary antibody, the sections were rinsed three times for $10 \mathrm{~min}$ in PBS and incubated in ImmPRESS Anti-Rabbit Immunoglobulin G Peroxidase-Linked Secondary Antibody (Vector Labs). Immunoreactivity was visualized using a DAB Substrate Kit (Vector Labs). After several rinses in PBS, the sections were mounted on coated glass slides, air-dried, dehydrated through a series of graded ethanol and clearing agent, and mounted with Permount.

Bright-field images of Fos immunoreactivity in the brain areas were captured using a charge-coupled device camera (CoolSNAP; Photometrics) and QImaging EXi Aqua attached to a Zeiss Axioskop 2 microscope. Images for counting labeled cells were captured at 20x magnification. Labeled cells from three sections per rat were bilaterally and automatically counted using IPLab 3.9.4 r5 software for Macintosh (Scanalytics) and iVision 4.0.15 software for Macintosh (BioVision). Counts from all images from each rat were averaged, so that each rat was an $n$ of 1 .

\section{Electrophysiology}

STN sections were prepared from naïve male Wistar rats (250-300 g) that were anesthetized with isoflurane (3\%) and decapitated. STN DBS was performed on the slice using the same parameters that were used for the in vivo experiments, with the exception of the duration of stimulation, which was reduced to either $1 \mathrm{~s}$ or $1 \mathrm{~min}$ (STN DBS longer than $1 \mathrm{~min}$ was associated with loss of the recorded cells due to micromovement of the slice). The brains were rapidly removed and placed into oxygenated $\left(95 \% \mathrm{O}_{2}\right.$ and $5 \%$ $\mathrm{CO}_{2} ; \mathrm{pH}$ 7.3) ice-cold high-sucrose solution containing (in $\mathrm{mM}$ ): sucrose 206; $\mathrm{KCl} 2.5 ; \mathrm{NaH}_{2} \mathrm{PO}_{4} 1.2 ; \mathrm{MgCl}_{2} 7 ; \mathrm{CaCl}_{2}$ $0.5 ; \mathrm{NaHCO}_{3}$ 26; glucose 5; HEPES 5. STN slices $(300 \mu \mathrm{m})$ were transferred into oxygenated artificial cerebrospinal fluid (aCSF) of the following composition (in $\mathrm{mM}$ ): $\mathrm{NaCl}, 130$; $\mathrm{KCl}, 3.5 ; \mathrm{NaH}_{2} \mathrm{PO}_{4}, 1.25 ; \mathrm{MgSO}_{4} \cdot 7 \mathrm{H}_{2} \mathrm{O}, 1.5 ; \mathrm{CaCl}_{2}, 2.0$; $\mathrm{NaHCO}_{3}, 24$; glucose, 10. Sections were first incubated for $30 \mathrm{~min}$ at $35-37^{\circ} \mathrm{C}$ and then kept at room temperature for the remainder of the experiment. We transferred individual sections to a recording chamber mounted on the stage of an upright microscope (Olympus BX50WI, Waltham MA, USA) for each experiment. Recordings were performed in continuously oxygenated aCSF perfused at a rate of $2-3 \mathrm{ml} / \mathrm{min}$.
Whole-cell configuration was performed using current clamp mode with a Multiclamp 700B amplifier, Digidata 1440A, and pClamp10 software (Molecular Devices, Sunnyvale, CA, USA) for recording. Neurons were visualized using a water immersion objective (Olympus), infrared differential interference contrast optics, and a CCD camera (EXi Aqua and ROLERA-XR, QImaging, Surrey, BC, Canada). Patch pipettes (impedance range 4-8 $M^{\prime} \Omega$ ) were pulled from borosilicate glass (Warner Instruments, Hamden, CT, USA) with a PC-10 puller (Narishige, East Meadow, NY, USA) and filled with the following internal solution (in $\mathrm{mM}$ ): $\mathrm{KMeSO}_{4} 70 ; \mathrm{KCl} 55 ; \mathrm{NaCl}$ 10; HEPES 10; $\mathrm{MgCl}_{2} 2 ; \mathrm{Mg}$-ATP 2; Na-GTP 0.2.

Stimulation paradigms: To study neuronal responses elicited by DBS, a concentric bipolar stimulation microelectrode (FHC, Bowdoin, ME, USA) was positioned in the middle of the STN. We used a SIU90 isolator (Neuro Data, New York NY) controlled by a Master-8 stimulator (AMPI, Jerusalem, Israel) to generate trains of HFS consisting of multiple single pulses $(60 \mu \mathrm{s}, 100 \mathrm{~m}$ delivered at a frequency of $130 \mathrm{~Hz}$ for a duration of $1 \mathrm{~s}$ or $1 \mathrm{~min}$.

\section{Statistical Analyses}

All of the data are expressed as means and SEM. Escalation data were analyzed by using one-way or mixed-factorial analysis of variance (ANOVA). When appropriate, post hoc comparisons were performed using the Tukey's multiple comparison test. Differences in breakpoints were analyzed between LgA and ShA groups for each dose using Student's $t$-test. Differences in quadrant crossings before and after STN HFS (prestimulation-poststimulation) were analyzed between groups using Student's $t$-test. Differences in estimated Fos populations between each structure were analyzed using Student's $t$-test. Finally, ANOVA was used to evaluate differences in firing rates from baseline, stimulation, and recovery followed by the Newman-Keuls post hoc test. For all of the tests, values of $p<0.05$ were considered statistically significant.

\section{RESULTS}

We first evaluated whether STN stimulation has an effect in an ShA ( $3 \mathrm{~h})$ model of heroin self-administration. Animals were trained in eight $1-h$ sessions before stimulation. Figure 2a shows the final five training sessions where animals developed stable lever pressing; there were no differences observed between groups. Once stable lever pressing was achieved, animals were then stimulated in $3 \mathrm{~h}$ sessions. The mixed-factorial ANOVA, with group as the between-subjects factor and time as the within-subjects factor, revealed significant effects of group $\left(\mathrm{F}_{1,18}=7.597\right.$, $p=0.0130)$ and time $\left(\mathrm{F}_{10,18}=4.106, p<0.0001\right)$ but no group $\times$ time interaction $\left(F_{10,180}=1.058, p>0.05\right)$. As shown in Figure 2b, rats with STN HFS self-administered less heroin compared with control rats during the entire period of STN HFS (group effect: $F_{1,18}=7.597, p=0.0130$ ). To examine the effects of STN HFS on reward efficacy or the motivation to work for the drug, the animals were tested in a PR test the day after the last FR self-administration session. As confirmed by the $t$-test analysis, rats with STN HFS 

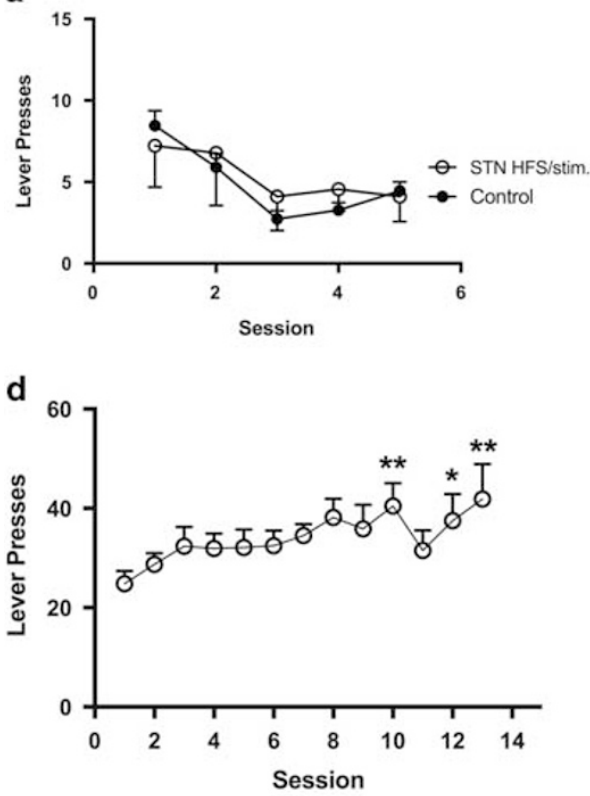

f

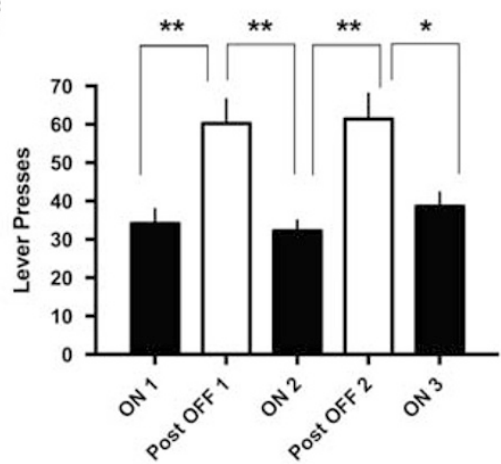

h

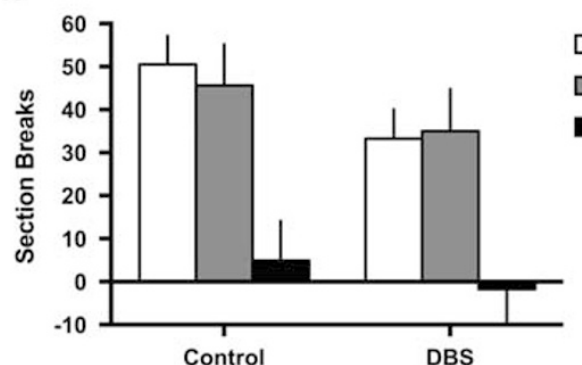

b
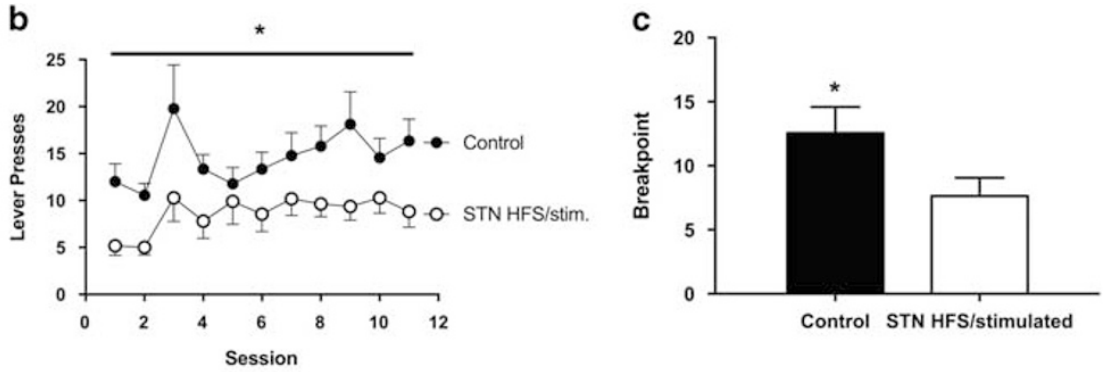

e

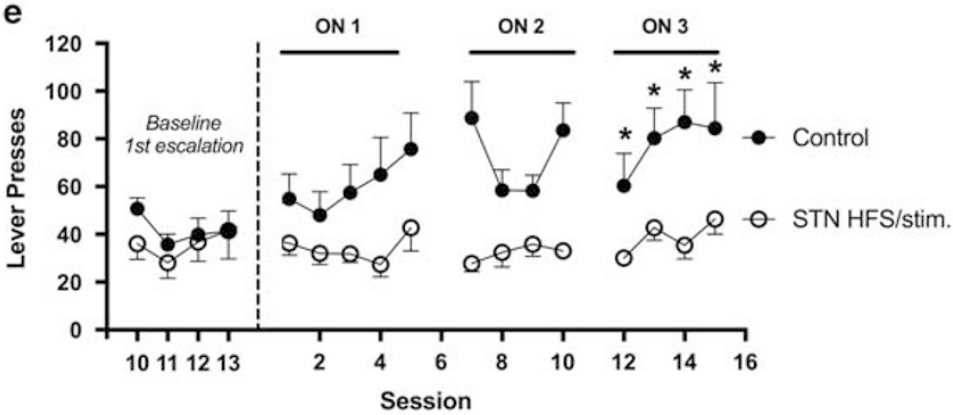

g

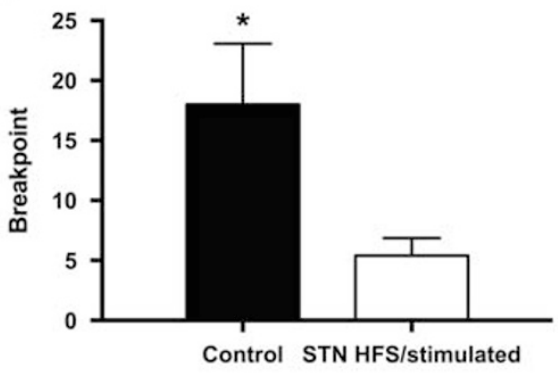

i

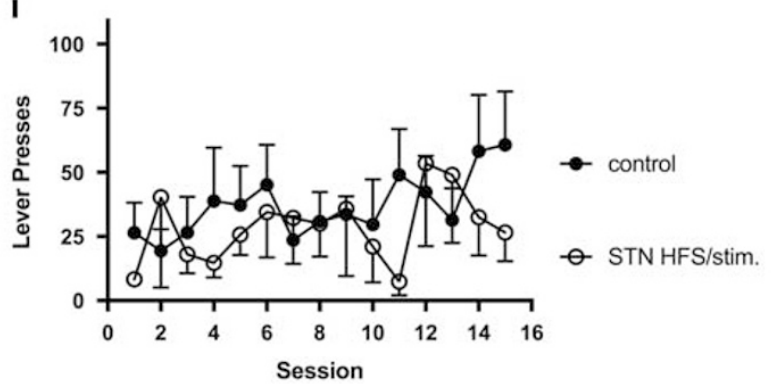

Figure 2 Heroin self-administration. Heroin self-administration during short-access sessions (Experiment I) is represented in panels a-c. (a) Training. Number of heroin infusions in I h training sessions (no stimulation) between subthalamic nucleus (STN) (black circles) and control animals (white circles). (b) Short access. Number of heroin infusions in 3-h sessions in animals that received high-frequency stimulation (HFS) of the STN (black circles) and control animals (white circles). $* p<0.05$, significant group effect. (c) Progressive ratio. Breakpoint reached after $3 \mathrm{~h}$ session in animals that received HFS in the STN (black bar) and control animals (white bar). ${ }^{*} p<0.05$, significant difference compared with control group. Heroin self-administration during long-access sessions (Experiment 2) is represented in panels $\mathrm{d}-\mathrm{g}$. (d) Initial escalation of heroin self-administration during fourteen I0-h sessions. Heroin self-administration is shown as average lever presses for all animals. ${ }^{*} p<0.05$, ${ }^{*} p<0.0$ I, significant difference compared with first day of long access. (e) Re-escalation during fifteen 10-h sessions in the stimulated (stim.) STN HFS and control groups. Sessions 5 and 10 were followed by a 2-day break from both stimulation and heroin access before the start of the subsequent session. $* p<0.05$, significant difference compared with control group. ( $f$ ) Effects on heroin consumption following 2-day breaks from stimulation in the STN HFS group. Phases I-3 (black bars) show heroin self-administration during stimulation periods. ON- I, average heroin self-administration for sessions 1-5; ON-2, average heroin self-administration for sessions 7-10; ON-3, average heroin self-administration for sessions 12-15. White bars indicate the days of non-stimulation. ${ }^{*} p<0.05$, $* * 2 p 0.00 \mathrm{I}$, significant differences compared with OFF days. (g) Progressive ratio shown as breakpoints reached after $6 \mathrm{~h}$ session in animals in the control group (white bar) and STN HFS group (black bar). *p $<0.05$, significant difference compared with control group. Motor effects from STN HFS in control and stimulated animals are represented in panels $h$ and i. (h) Animals were assessed for differences in locomotor activity by observing section breaks in their operant boxes before and during stimulation. (i) Lever pressing on the inactive lever during the self-administration sessions were recorded and compared between groups. Error bars indicate SEM. DBS, deep brain stimulation. 
had significantly lower breakpoints than control rats $\left(t_{18}=2.043, p=0.05\right.$; Figure $\left.2 c\right)$.

We next tested the effects of stimulation in a re-escalation model of heroin self-administration. In this experiment, all of the animals in long-access (12 h) sessions exhibited an increase in heroin intake over the course of 13 sessions (heroin intake effect: $\mathrm{F}_{14,168}=16.80, p<0.0001$ ). Tukey's multiple comparison test showed that animals exhibited escalated heroin intake on days 10,12 , and $13(p<0.05$ and $p<0.01$ compared with day 1; Figure $2 \mathrm{~d}$ ). The animals were then given 2 weeks of abstinence and separated into two groups that were matched for heroin intake: one group received STN HFS during the re-escalation period and another group served as non-stimulated control animals. The mixed-factorial ANOVA, with group as the between-subjects factor and time as the within-subjects factor, revealed significant effects of group $\left(\mathrm{F}_{1,10}=7.700, p=0.0196\right)$ and time $\left(\mathrm{F}_{12,10}=2.965, p=0.0012\right)$ and a group $\times$ time interaction $\left(\mathrm{F}_{12,120}=1.829, p=0.05\right)$. Tukey's multiple comparison test revealed that the STN HFS group exhibited lower heroin intake on days $12,13,14$, and 15 of the re-escalation period (all $p<0.05$; Figure 2e).

To test the reversibility of STN HFS, we measured heroin self-administration before (ON-1) and after 2 days of nonstimulation (OFF), followed by an additional 4 days of stimulation (ON-2) in a within-subjects design. The overall ANOVA revealed a significant effect of stimulation $\left(\mathrm{F}_{4,20}=8.581, p=0.0003\right)$. Tukey's multiple comparison test showed that following each 2-day break (OFF-1, session 6 and OFF-2, session 11) from STN HFS, the animals significantly increased heroin intake $(p<0.05$ and $p<0.01$; Figure 2f).

After the end of the escalation period, the rats were tested in a PR test. Control rats had significantly higher breakpoints $(t(10)=2.459, p=0.0338)$ compared with stimulated rats (Figure 2g), suggesting that the HFS animals were less motivated to lever press for heroin compared with animals in the control group. Finally, we examined potential differences in locomotor activity by assessing section breaks and pressing on the inactive lever. No differences were found in locomotor activity (section breaks) before or during stimulation (difference score between groups: $p=0.6599$; Figure 2h) or in inactive lever pressing $(p=0.7841$; Figure 2i), indicating that the decrease in heroin intake was not attributable to a decrease in general activity.

To evaluate the effect of STN HFS on the neuronal network associated with the STN and brain regions associated with drug addiction, we measured the number of Fos-positive neurons as an indicator of activation following stimulation. We found that stimulation of the STN significantly decreased the number of Fos-positive neurons in two output regions of the STN (substantia nigra pars reticulata [SNr], $p=0.0045$; entopeduncular nucleus [EP] $p=0.0149 ;$ nucleus accumbens shell [NAcSh], $p=0.0284$; Figure $3 \mathrm{~m}$ ). A strong trend toward an increase in Fos-positive neurons was observed in the prefrontal cortex between the stimulated and control animals (Figure $3 \mathrm{~m}$ ). We also examined the insula, anteroventral thalamus, and ventral tegmental area (VTA), all of which showed no difference (data not shown).

To evaluate neuronal activity in the STN following HFS, we assessed the effects of stimulation on spontaneous

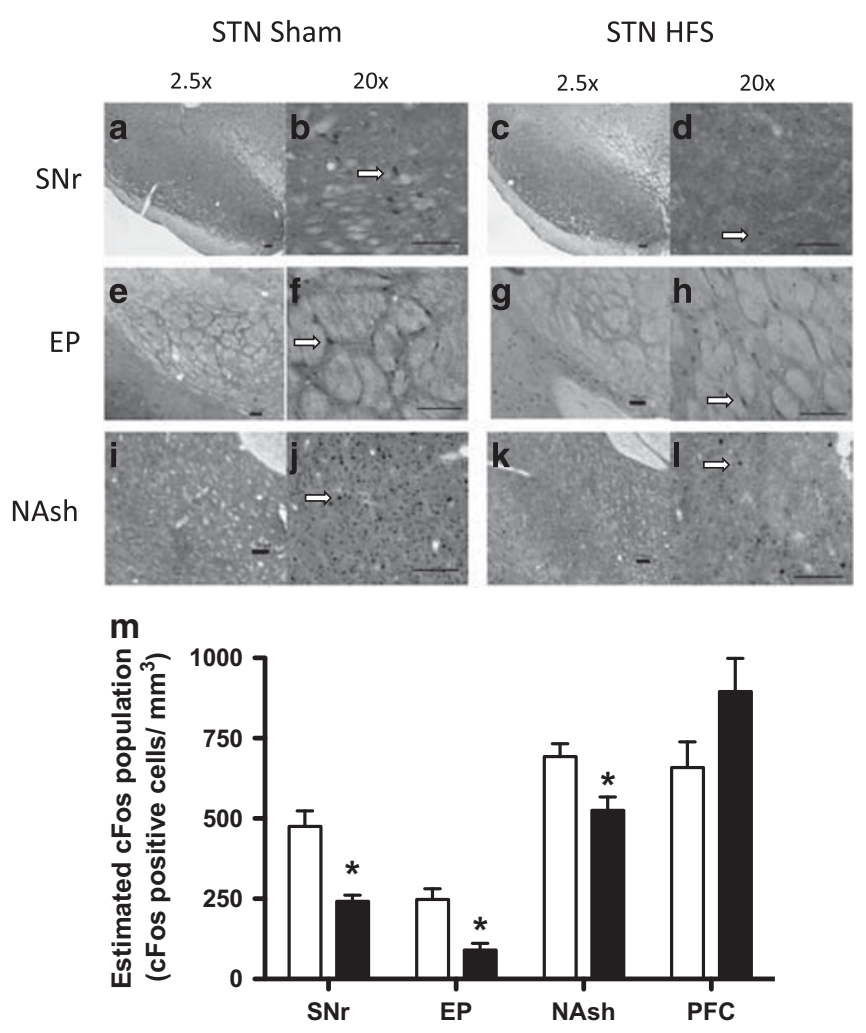

Figure 3 Fos expression in control and stimulated animals. The brain areas that were examined were the substantia nigra pars reticulate $(\mathrm{SNr})$ $(a-d)$, entopeduncular nucleus (EP) (e-h), and nucleus accumbens shell (NAsh) (i-l). Left panels for each structure show images that were taken from control rats, and right panels show images that were taken from stimulated rats. Orientation is noted at $2.5 \times$ magnification (left panels), and cell counts were taken at $20 \times$ magnification (right panels). Arrows highlight a typical Fos-positive neuron for each section. Scale bars $=100 \mu \mathrm{m}$. (m) The quantification of Fos expression in each structure is illustrated for control rats (empty bars) and subthalamic nucleus high-frequency stimulation (STN HFS) rats (black bars) and was calculated using IPLab 3.9.4 r5 software for Macintosh (Scanalytics) and iVision 4.0.I 5 software for Macintosh (BioVision). ${ }^{*} p<0.05$, significant effect compared with control group. Error bars indicate SEM. PFC, prefrontal cortex.

neuronal activity with the stimulation parameters used in the behavioral study. After establishing stable recording conditions (3-5 min after break-in), STN neurons were manually depolarized to elicit firing of action potentials (APs; threshold $-52 \pm 0.4 \mathrm{mV}$ ). The AP firing frequency of STN neurons was quantified for at least $60 \mathrm{~s}$ just before HFS delivery (baseline), then during the silencing period that followed the train of stimulations (Stim) and after recovery (Recv) to steady AP firing. We first assessed the effects of a 1-s train of stimulations on spontaneous activity. Immediately following HFS delivery, neurons slightly hyperpolarized and AP firing was decreased (Figure 4a), displaying a 'silencing' period. On average, AP firing decreased from $1.43 \pm 0.16$ to $0.52 \pm 0.19 \mathrm{~Hz}$ following delivery of a 1 -s HFS train $(n=7$; Figure $4 \mathrm{~b})$. At the end of the silencing period elicited by HFS, spontaneous activity recovered to a level comparable to that observed before HFS $(1.52 \pm 0.14 \mathrm{~Hz})$. Overall ANOVA showed a significant effect of a 1-s HFS $\left(\mathrm{F}_{2,6}=22.04, p<0.001\right)$. The Newman-Keuls post hoc analysis confirmed that compared with baseline, the firing 
a

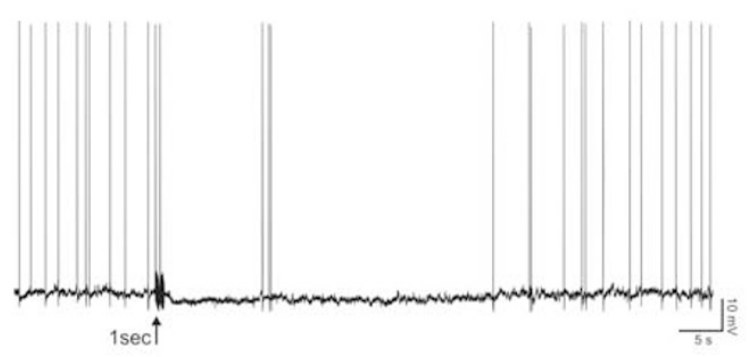

e

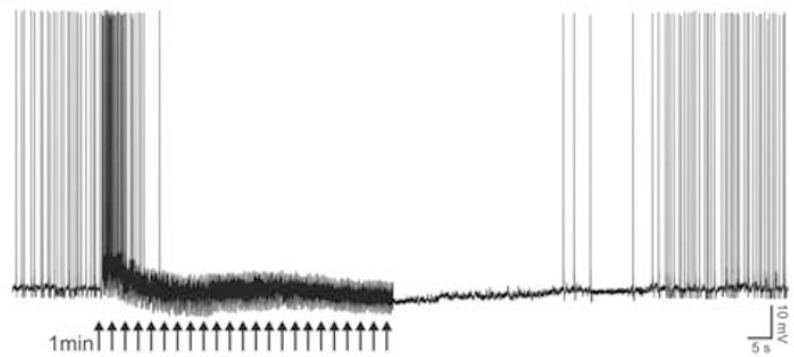

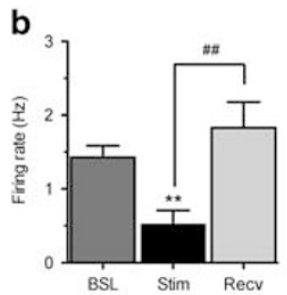

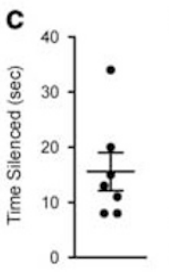

d
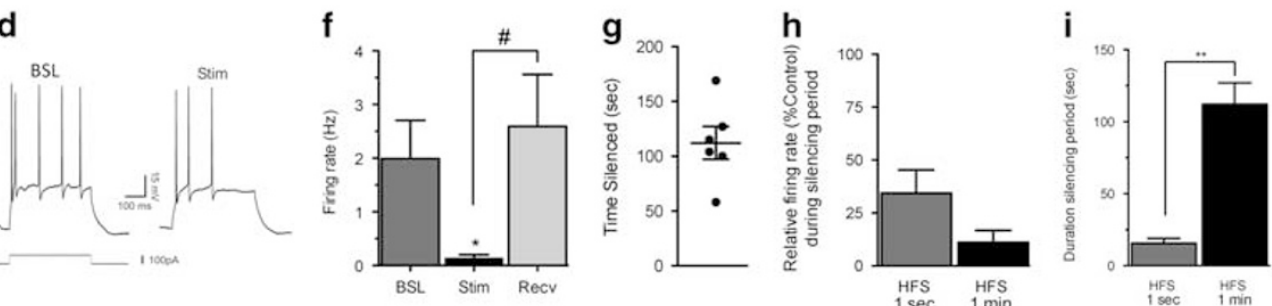

Figure 4 Subthalamic nucleus (STN) activity following high-frequency stimulation (HFS). STN activity following a I-s stimulation is represented in (a-d). (a) Continuous intracellular recording of a STN neuron in current-clamp mode. Tonic firing was depressed following a I-s HFS (arrow), and the silencing period lasted 34 s until recovery to pre-HFS level. (b) On average, the action potential (AP) firing was significantly decreased from I.43 to $0.52 \mathrm{~Hz}$, with recovery to $1.52 \mathrm{~Hz}(n=7)$. $* * 0<0.01$ indicates significant effect of stimulation compared with baseline. ${ }^{\# \#} p<0.0$ I shows significant effect of recovery compared with stimulation. Error bars indicate SEM. (c) Following delivery of a I-s HFS, the silencing period lasted $16 \mathrm{~s}(n=7)$. (d) In another neuron, injection of a depolarizing current step before HFS (Control) triggered five APs, and the same current step delivered at the same holding potential during the silencing period (Stim) triggered only three APs. STN activity following a I-min stimulation is represented in (e-i). (e) Continuous recording of a STN neuron. Delivery of a I-min HFS (multiple arrows) first increased tonic firing for a few seconds and then markedly inhibited neuronal activity for $105 \mathrm{~s}$ until recovery to pre-HFS level. ( $f$ ) On average, the AP firing was significantly decreased from 1.99 to $0.13 \mathrm{~Hz}$, with recovery to $2.59 \mathrm{~Hz}(n=6)$. ${ }^{2} p<0.05$ indicates significant effect of stimulation compared with baseline. ${ }^{\#} p<0.05$ shows significant effect of recovery compared with stimulation. Error bars indicate SEM. (g) Upon delivery of a I-min HFS, the silencing period lasted II $\mathrm{s}(n=6)$. (h) The depressing effect of HFS on neuronal activity was more pronounced with a I-min duration (89\% decrease) compared with I-s (66\% decrease). (i) The duration of the silencing period was significantly longer with a I-min HFS compared with a I-s HFS. *** $<0.0$ I indicates significant effect of I-min stimulation compared with I-s stimulation. Error bars indicate SEM. BSL, baseline; Recv, recovery; Stim, stimulations.

rate was significantly lower during the silencing period $(p<0.01)$. Following the silencing period, the firing rate recovered to a level comparable to baseline level $(p<0.01$ from Stim; Figure $4 \mathrm{~b}$ ). The depression of firing was transient and lasted on average $15.6 \pm 3.5 \mathrm{~s}$ (Figure 4c). We also assessed AP firing evoked with depolarizing current pulses just prior to and immediately following HFS. The number of APs elicited by a depolarizing step delivered at similar holding potentials was decreased during the silencing period (Figure 4d), suggesting an alteration of neuronal intrinsic properties by HFS.

We next studied the effect elicited by delivery of a continuous 1-min HFS. The spontaneous firing decreased within 3-7 s of initiating the train of stimulations, followed by a marked silencing of neuronal activity (Figure 4e). Note that some neurons showed increased AP firing for a few seconds (seen in Figure 4e), perhaps because more proximal stimulation sites and summation of synaptic responses in these instances. On average, AP firing decreased from $1.99 \pm 0.66 \mathrm{~Hz}$ before HFS to $0.13 \pm 0.06 \mathrm{~Hz}$ in the silencing period that started during delivery of the 1-min HFS, recovering to $2.59 \pm 0.90 \mathrm{~Hz}$ ( $n=6$; Figure $4 \mathrm{f})$. The overall ANOVA showed a significant effect of HFS $\left(\mathrm{F}_{2,5}=3.213\right.$, $p<0.05)$. The Newman-Keuls post hoc analyses confirmed that compared with baseline, the firing rate was significantly lower after the HFS $(p<0.05)$. Following the silencing period, the firing rate recovered to a level that was not significantly different from baseline $(p<0.01$ from Stim; Figure 4f). The decrease in AP firing lasted for $112 \pm 14 \mathrm{~s}$
(Figure 4g). Assessment of the number of APs elicited by a depolarizing pulse in a few neurons suggested decreased intrinsic excitability of STN neurons immediately following a 1-min HFS (not shown).

Overall, expression of the change in firing rate relative to baseline values showed a more pronounced effect with the 1min HFS $(11 \pm 5 \%$ of control) compared with the 1-s HFS $(34 \pm 11 \%$ of control; Figure $4 \mathrm{~h})$, but this effect did not reach significance $\left(t_{11}=1.78 ; p>0.05\right)$. The duration of silencing, however, was significantly longer with the 1-min HFS compared with the 1-s HFS $\left(t_{11}=6.83, p<0.01\right)$ as revealed by unpaired Student's $t$-test analysis (Figure 4i). We could not assess the effect of a longer duration of stimulation (eg, $30 \mathrm{~min}$ ) using this ex vivo preparation because longer stimulation resulted in the loss of the membrane seal with the recording electrode.

\section{DISCUSSION}

The present study provides preclinical evidence of the potential therapeutic efficacy of STN HFS for heroin addiction. The results showed that STN HFS decreased heroin self-administration in ShA sessions and prevented the re-escalation of heroin self-administration after abstinence in long-access sessions. We also showed that STN HFS produced a robust silencing of STN neurons and decreased neuronal activity in the SNr and EP, two output regions of 
the STN, and in the NAcSh, a key region in the control of motivated behavior.

This report demonstrates that STN HFS has the ability to prevent increases in escalation following a period of abstinence. The escalation model that was used in the present study has been shown to produce many independent markers of opiate addiction, including the escalation of intake, increases in PR breakpoints, increases in somatic withdrawal, and increases in intake after a period of abstinence (re-escalation) (Barbier et al, 2013). Although control animals showed pronounced re-escalation of heroin intake, with rats doubling their intake after 2 weeks of abstinence, rats that received STN HFS showed a steady rate of lever pressing during the re-escalation phase of this experiment, without any increase in heroin selfadministration. The re-escalation patterns that were observed in the control animals are indicative of the transition from controlled drug intake to compulsive-like drug taking. The fact that LgA rats were even more sensitive to the effects of STN HFS than ShA rats in the PR test and that STN HFS attenuated re-escalation after abstinence from escalated heroin intake demonstrate the potential for STN HFS to be used as an alternative therapy for heroin addictions in populations with heavy compulsive heroin intake, including those who experience repeated failures to remain abstinent or who are resistant to pharmacotherapies.

Studies examining brain reward thresholds following ShA or $\operatorname{LgA}$ sessions support the hypothesis that ShA selfadministration can increase reward sensitivity, whereas LgA self-administration leads to decreased reward sensitivity, resulting in increased daily intake (Kenny et al, 2006). Considering that lesions of the STN in rats reduces affective responses to both positive reinforcers (eg, sweet solution) and negative reinforcers (eg, electric footshock or lithium chloride-induced sickness) (Pelloux et al, 2014), one may hypothesize that the decrease in heroin self-administration that was observed in ShA and LgA rats is mediated by a reduction of both the positive reinforcing effect of heroin and a decrease in the negative emotional state of withdrawal. Evidence in human studies further supports this hypothesis. Indeed, STN HFS in humans has been shown to impair the ability to correctly identify facial expressions of negative emotions (Geday et al, 2006; Le Jeune et al, 2010a). While this presents a challenge and may add to behavioral problems, it has been noted that patients with social maladjustments may benefit from tempered emotional states (Geday et al, 2006). Lesions of the STN shifted preference from a more pleasant, non-caloric reward (ie, saccharin) to a caloric less-pleasant reward (glucose) (Pelloux et al, 2014), suggesting that STN HFS reduces the affective properties of rewards.

The interruption of STN HFS for 2 days was associated with a robust increase in heroin self-administration to levels that were similar to control rats. The resumption of STN HFS following temporary interruption reduced lever pressing to prior levels. These results show that the effect of STN HFS on heroin self-administration was reversible, further confirming the causal effect of STN HFS on heroin selfadministration and suggesting that chronic STN HFS may be required to observe clinically relevant effects. This hypothesis is supported by previous work that was performed in rats, showing that switching off STN HFS reverses the effects of
STN HFS on cocaine motivation/seeking (Rouaud et al, 2010). The results are consistent with many reports that have demonstrated the reversibility of the effects of STN HFS in Parkinson's disease (Henderson and Dunnett, 1998; Pollak et al, 2002).

The mechanism that underlies the beneficial effects of STN HFS for the treatment of Parkinson's disease is unclear because both the inactivation and activation of target structures have been reported (Garcia et al, 2003; Hashimoto et al, 2003). Although STN HFS may transiently excite STN neurons, converging evidence suggests that STN HFS leads to the sustained suppression of spontaneous activity of STN neurons (Beurrier et al, 2001; Lee et al, 2003; Magarinos-Ascone et al, 2002) combined with the generation of a new pattern of activity that is time locked to the stimulation pattern. These results provide a cellular mechanism for the clinical effect of STN HFS in Parkinson's disease, in which STN HFS may silence abnormal bursting patterns of discharge in output regions of the basal ganglia $(\mathrm{SNr}$ and EP) associated with the motor symptoms of Parkinson's disease.

Both our cellular recording and Fos brain mapping results confirm this hypothesis and further demonstrate that STN HFS decreases the activity of STN neurons even at levels of stimulation that are below the threshold of altering locomotor activity. Our electrophysiological results show that delivery of a 1-s HFS in the STN depresses spontaneous neuronal activity. This silencing effect rapidly developed and was reversible after cessation of the stimulation. The magnitude and duration of silencing of STN neurons observed upon HFS was extended by increasing the HFS duration to $1 \mathrm{~min}$, and the recovery to control firing level could still be obtained within $60 \mathrm{~s}$ after the end of the HFS. Such duration of effect following cessation of stimulation is short lasting, implying that sustained ongoing stimulation such as those delivered in our behavioral study $(130 \mathrm{~Hz}$ frequency and $60 \mu$ s pulse width) are necessary to achieve consistent and reliable silencing of STN neurons over extended periods of time in vivo.

Previous ex vivo electrophysiological studies have shown that stimulation of the STN in rat slices can result in inhibition of neuronal excitability during and after cessation of the stimulation (Beurrier et al, 2001; Garcia et al, 2003; Lee et al, 2003; Magarinos-Ascone et al, 2002), consistent with our results. Such results have also been obtained in rat in vivo (Benazzouz et al, 1995; Tai et al, 2003) and while recording human neuronal activity (Filali et al, 2004). The stimulus intensity and frequency $(0.1 \mathrm{~mA}, 130 \mathrm{~Hz})$ that we used were conservative compared with previous studies, but the degree of inhibition of AP firing compared well to previous studies (Beurrier et al, 2001; Garcia et al, 2003; Lee et al, 2003; Magarinos-Ascone et al, 2002). We observed that neuronal excitability assessed with depolarizing current steps was decreased, suggesting a postsynaptic effect on intrinsic neuronal properties. Previous work in the STN has shown that a 1-min HFS depresses neuronal activity by altering sodium and calcium channel activity (Beurrier et al, 2001). Based on our results and these previous reports, it is likely that HFS inhibits STN neurons by acting at a postsynaptic site and results in inhibition of output structures ( $\mathrm{SNr}, \mathrm{EP}$ ).

Consistent with this hypothesis, the brain mapping results showed that STN HFS decreased the number of Fos-positive 
neurons in the SNr and EP, suggesting that STN HFS decreased the overall output of the STN. It is important to note that this experiment was carried out in the absence of heroin self-administration to eliminate the confounding effect of differential heroin intake in control vs STN HFS animals. Indeed, animals that received STN HFS selfadministered less heroin compared with controls, and it would not have been possible to dissociate the effect of STN HFS $v s$ the effect of lower heroin intake on neuronal activity in the brain regions of interest. The present results are consistent with previous studies that reported decreases in metabolic activity (Benazzouz et al, 1995; Tai et al, 2003) and decreases in immediate-early gene levels (Creed et al, 2012) in the STN's output regions after STN HFS. Our results do not confirm previous reports that showed either no change or increases in Fos-positive neurons in the SNr and EP after STN HFS (Hachem-Delaunay et al, 2015; Shehab et al, 2014; Tan et al, 2011). Different techniques (ie, IHC $v s$ in situ hybridization) and different times of stimulation $v s$ sacrifice may explain these discrepancies. Our protocol may have allowed detection of the long-term inhibitory effect of STN HFS on cell bodies, whereas previous protocols may have only allowed the detection of short-term fiber stimulation (Magarinos-Ascone et al, 2002). Because of glutamatergic projections from the STN, inhibition of these neurons should lead to a reduction of activity of output structures (ie, $\mathrm{SNr}$ and EP). Microdialysis studies have shown increased levels of dopamine in the striatum after STN HFS (Winter et al, 2008). In contrast, STN HFS reduced cocaine-induced Fos expression in the NAc (Hachem-Delaunay et al, 2015). No changes were observed in the NAc core, insula, anteroventral thalamus, or VTA.

We observed a decrease in the number of Fos-positive neurons in the NAcSh after STN HFS, confirming the possibility that modulation of the NAcSh by STN HFS may have a key role in the therapeutic-like effects of STN HFS. A strong trend toward an increase in the number of Fospositive neurons was observed in the prefrontal cortex after STN HFS. This increase, suggesting increased activity in the prefrontal cortex after STN HFS, has been reported previously (Tan et al, 2011), but contrasts with positron emission tomography studies that reported decreased metabolic activity in the prefrontal cortex after STN HFS in obsessive-compulsive disorder patients (Le Jeune et al, 2010b). The increased activity in the PFC observed here may be expected when considering the existence of the hyperdirect pathway between the prefrontal cortex and STN and the possibility of the antidromic stimulation of pyramidal neurons that is caused by STN HFS. The STN is a key structure in the basal ganglia that sends excitatory projections to the $\mathrm{SNr}$ and EP. Our results show that STN HFS inactivated two main output regions (ie, the EP and $\mathrm{SNr}$ ), suggesting that the therapeutic effect of STN HFS on heroin dependence may be produced by sustained inhibition of the SNr, EP, and NAcSh.

The present study provides robust preclinical evidence of the potential therapeutic efficacy of STN HFS for heroin addiction using an animal model of compulsive heroin seeking. The results further suggest a mechanism of action for STN HFS that involves inhibition of the SNr, EP, and NAcSh, leading to a decrease in the motivation for heroin and the prevention of re-escalation of heroin intake after abstinence. These results strongly suggest that STN HFS may have a beneficial effect on preventing the re-engagement of compulsive drug seeking of heroin and provide a strong rationale for investigating these effects in human studies.

\section{FUNDING AND DISCLOSURE}

Research was financially supported by National Institutes of Health Grants DA DA029821 from the National Institute on Drug Abuse and the Pearson Center for Alcoholism and Addiction Research. The authors declare no conflict of interest.

\section{ACKNOWLEDGMENTS}

We thank Michael Arends for proofreading the manuscript.

\section{REFERENCES}

Ardouin C, Voon V, Worbe Y, Abouazar N, Czernecki V, Hosseini $\mathrm{H}$ et al (2006). Pathological gambling in Parkinson's disease improves on chronic subthalamic nucleus stimulation. Movement Disord 21: 1941-1946.

Barbier E, Vendruscolo LF, Schlosburg JE, Edwards S, Juergens N, Park PE et al (2013). The NK1 receptor antagonist L822429 reduces heroin reinforcement. Neuropsychopharmacology 38: 976-984.

Baunez C, Dias C, Cador M, Amalric M (2005). The subthalamic nucleus exerts opposite control on cocaine and 'natural' rewards. Nat Neurosci 8: 484-489.

Benazzouz A, Piallat B, Pollak P, Benabid AL (1995). Responses of substantia nigra pars reticulata and globus pallidus complex to high frequency stimulation of the subthalamic nucleus in rats: electrophysiological data. Neurosci Lett 189: 77-80.

Beurrier C, Bioulac B, Audin J, Hammond C (2001). Highfrequency stimulation produces a transient blockade of voltagegated currents in subthalamic neurons. J Neurophysiol 85: 1351-1356.

Chen SA, O'Dell LE, Hoefer ME, Greenwell TN, Zorrilla EP, Koob GF (2006). Unlimited access to heroin self-administration: independent motivational markers of opiate dependence. Neuropsychopharmacology 31: 2692-2707.

Crane EH (2015). The CBHSQ Report: Emergency Department Visits Involving Narcotic Pain Relievers. Substance Abuse and Mental Health Services Administration, Center for Behavioral Health Statistics and Quality: Rockville, MD.

Creed MC, Hamani C, Nobrega JN (2012). Early gene mapping after deep brain stimulation in a rat model of tardive dyskinesia: comparison with transient local inactivation. Eur Neuropsychopharmacol 22: 506-517.

Darbaky Y, Forni C, Amalric M, Baunez C (2003). High frequency stimulation of the subthalamic nucleus has beneficial antiparkinsonian effects on motor functions in rats, but less efficiency in a choice reaction time task. Eur J Neurosci 18: 951-956.

Filali M, Hutchison WD, Palter VN, Lozano AM, Dostrovsky JO (2004). Stimulation-induced inhibition of neuronal firing in human subthalamic nucleus. Exp Brain Res 156: 274-281.

Garcia L, Audin J, D'Alessandro G, Bioulac B, Hammond C (2003). Dual effect of high-frequency stimulation on subthalamic neuron activity. J Neurosci 23: 8743-8751.

Geday J, Ostergaard K, Gjedde A (2006). Stimulation of subthalamic nucleus inhibits emotional activation of fusiform gyrus. NeuroImage 33: 706-714.

Guercio LA, Schmidt HD, Pierce RC (2015). Deep brain stimulation of the nucleus accumbens shell attenuates cue-induced 
reinstatement of both cocaine and sucrose seeking in rats. Behav Brain Res 281: 125-130.

Guo L, Zhou H, Wang R, Xu J, Zhou W, Zhang F et al (2013). DBS of nucleus accumbens on heroin seeking behaviors in self-administering rats. Drug Alcohol Depend 129: 70-81.

Hachem-Delaunay S, Fournier ML, Cohen C, Bonneau N, Cador M, Baunez C et al (2015). Subthalamic nucleus high-frequency stimulation modulates neuronal reactivity to cocaine within the reward circuit. Neurobiol Dis 80: 54-62.

Hamani C, Pilitis J, Rughani A, Rosenow J, Patil P, Slavin K et al (2014). Deep brain stimulation for obsessive-compulsive disorder: systematic review and evidence-based guideline sponsored by the American Society for Stereotactic and Functional Neurosurgery and the Congress of Neurological Surgeons (CNS) and endorsed by the CNS and American Association of Neurological Surgeons. Neurosurgery 74: 327-333.

Hashimoto T, Elder CM, Okun MS, Patrick SK, Vitek JL (2003). Stimulation of the subthalamic nucleus changes the firing pattern of pallidal neurons. J Neurosci 23: 1916-1923.

Henderson JM, Dunnett SB (1998). Targeting the subthalamic nucleus in the treatment of Parkinson's disease. Brain Res Bull 46: 467-474.

Kenny PJ, Chen SA, Kitamura O, Markou A, Koob GF (2006). Conditioned withdrawal drives heroin consumption and decreases reward sensitivity. J Neurosci 26: 5894-5900.

Kuhn J, Moller M, Treppmann JF, Bartsch C, Lenartz D, Gruendler TO et al (2014). Deep brain stimulation of the nucleus accumbens and its usefulness in severe opioid addiction. Mol Psychiatry 19: 145-146.

Le Jeune F, Peron J, Grandjean D, Drapier S, Haegelen C, Garin E et al (2010a). Subthalamic nucleus stimulation affects limbic and associative circuits: a PET study. Eur J Nucl Med Mol Imag 37: $1512-1520$.

Le Jeune F, Verin M, N'Diaye K, Drapier D, Leray E, Du Montcel ST et al (2010b). Decrease of prefrontal metabolism after subthalamic stimulation in obsessive-compulsive disorder: a positron emission tomography study. Biol Psychiatry 68: 1016-1022.

Lee KH, Roberts DW, Kim U (2003). Effect of high-frequency stimulation of the subthalamic nucleus on subthalamic neurons: an intracellular study. Stereotactic Funct Neurosurg 80: $32-36$.

Magarinos-Ascone C, Pazo JH, Macadar O, Buno W (2002). High-frequency stimulation of the subthalamic nucleus silences subthalamic neurons: a possible cellular mechanism in Parkinson's disease. Neuroscience 115: 1109-1117.

Mallet L, Mesnage V, Houeto JL, Pelissolo A, Yelnik J, Behar C et al (2002). Compulsions, Parkinson's disease, and stimulation. Lancet 360: 1302-1304.

Mallet L, Polosan M, Jaafari N, Baup N, Welter ML, Fontaine D et al (2008). Subthalamic nucleus stimulation in severe obsessivecompulsive disorder. N Engl J Med 359: 2121-2134.

McLellan AT, Lewis DC, O'Brien CP, Kleber HD (2000). Drug dependence, a chronic medical illness: implications for treatment, insurance, and outcomes evaluation. JAMA 284: 1689-1695.

Paxinos G, Watson C, Pennisi M, Topple A (1985). Bregma, lambda and the interaural midpoint in stereotaxic surgery with rats of different sex, strain and weight. J Neurosci Methods 13: 139-143.
Pelloux Y, Meffre J, Giorla E, Baunez C (2014). The subthalamic nucleus keeps you high on emotion: behavioral consequences of its inactivation. Front Behav Neurosci 8: 414.

Pollak P, Fraix V, Krack P, Moro E, Mendes A, Chabardes S et al (2002). Treatment results: Parkinson's disease. Movement Disord 17(Suppl 3): S75-S83.

Rouaud T, Lardeux S, Panayotis N, Paleressompoulle D, Cador M, Baunez C (2010). Reducing the desire for cocaine with subthalamic nucleus deep brain stimulation. Proc Natl Acad Sci USA 107: 1196-1200.

Schlosburg JE, Vendruscolo LF, Bremer PT, Lockner JW, Wade CL, Nunes AA et al (2013). Dynamic vaccine blocks relapse to compulsive intake of heroin. Proc Natl Acad Sci USA 110: 9036-9041.

Shehab S, D'Souza C, Ljubisavljevic M, Redgrave P (2014). Highfrequency electrical stimulation of the subthalamic nucleus excites target structures in a model using c-fos immunohistochemistry. Neuroscience 270: 212-225.

Smyth B, Hoffman V, Fan J, Hser YI (2007). Years of potential life lost among heroin addicts 33 years after treatment. Prev Med 44: 369-374.

Stephen JH, Halpern CH, Barrios CJ, Balmuri U, Pisapia JM, Wolf JA et al (2012). Deep brain stimulation compared with methadone maintenance for the treatment of heroin dependence: a threshold and cost-effectiveness analysis. Addiction 107: 624-634.

Tai CH, Boraud T, Bezard E, Bioulac B, Gross C, Benazzouz A (2003). Electrophysiological and metabolic evidence that highfrequency stimulation of the subthalamic nucleus bridles neuronal activity in the subthalamic nucleus and the substantia nigra reticulata. FASEB J 17: 1820-1830.

Tan SK, Janssen ML, Jahanshahi A, Chouliaras L, VisserVandewalle V, Lim LW et al (2011). High frequency stimulation of the subthalamic nucleus increases c-fos immunoreactivity in the dorsal raphe nucleus and afferent brain regions. J Psychiatric Res 45: 1307-1315.

Valencia-Alfonso CE, Luigies J, Smolders R, Cohen MX, Levar N, Mazaheri A et al (2012). Effective deep brain stimulation in heroin addiction: a case report with complementary intracranial electroencephalogram. Biol Psychiatry 71: e35-e37.

Vassoler FM, Schmidt HD, Gerard ME, Famous KR, Ciraulo DA, Kornetsky C et al (2008). Deep brain stimulation of the nucleus accumbens shell attenuates cocaine priming-induced reinstatement of drug seeking in rats. J Neurosci 28: 8735-8739.

Vassoler FM, White SL, Hopkins TJ, Guercio LA, Espallergues J, Berton $O$ et al (2013). Deep brain stimulation of the nucleus accumbens shell attenuates cocaine reinstatement through local and antidromic activation. $J$ Neurosci 33: 14446-14454.

Vendruscolo LF, Schlosburg JE, Misra KK, Chen SA, Greenwell TN, Koob GF (2011). Escalation patterns of varying periods of heroin access. Pharmacol Biochem Behav 98: 570-574.

Winter C, Lemke C, Sohr R, Meissner W, Harnack D, Juckel G et al (2008). High frequency stimulation of the subthalamic nucleus modulates neurotransmission in limbic brain regions of the rat. Exp Brain Res 185: 497-507.

Witjas T, Baunez C, Henry JM, Delfini M, Regis J, Cherif AA et al (2005). Addiction in Parkinson's disease: impact of subthalamic nucleus deep brain stimulation. Movement Disord 20: 1052-1055.

Zhou H, Xu J, Jiang J (2011). Deep brain stimulation of nucleus accumbens on heroin-seeking behaviors: a case report. Biol Psychiatry 69: e41-e42. 